\title{
Sex differences in variability across timescales in BALB/c mice
}

\author{
Benjamin L. Smarr ${ }^{1}$, Azure D. Grant ${ }^{1}$, Irving Zucker ${ }^{1,2}$, Brian J. Prendergast ${ }^{3}$ and Lance J. Kriegsfeld ${ }^{1,4^{*}}$
}

\begin{abstract}
Background: Females are markedly underinvestigated in the biological and behavioral sciences due to the presumption that cyclic hormonal changes across the ovulatory cycle introduce excess variability to measures of interest in comparison to males. However, recent analyses indicate that male and female mice and rats exhibit comparable variability across numerous physiological and behavioral measures, even when the stage of the estrous cycle is not considered. Hormonal changes across the ovulatory cycle likely contribute cyclic, intra-individual variability in females, but the source(s) of male variability has, to our knowledge, not been investigated. It is unclear whether male variability, like that of females, is temporally structured and, therefore, quantifiable and predictable. Finally, whether males and females exhibit variability on similar time scales has not been explored.

Methods: These questions were addressed by collecting chronic, high temporal resolution locomotor activity (LA) and core body temperature (CBT) data from male and female BALB/c mice.

Results: Contrary to expectation, males are more variable than females over the course of the day (diel variability) and exhibit higher intra-individual daily range than females in both LA and CBT. Between mice of a given sex, variability is comparable for LA but the inter-individual daily range in CBT is greater for males. To identify potential rhythmic processes contributing to these sex differences, we employed wavelet transformations across a range of periodicities (1-39 h).

Conclusions: Although variability in circadian power is comparable between the sexes for both LA and CBT, infradian variability is greater in females and ultradian variability is greater in males. Thus, exclusion of female mice from studies because of estrous cycle variability may increase variance in investigations where only male measures are collected over a span of several hours and limit generalization of findings from males to females.
\end{abstract}

Keywords: Temporal structure, Longitudinal data, Biological rhythms, Ovulation, Estrous cycles, Wavelets, Time series analysis

\section{Background}

When exploring questions in fields other than reproductive biology, it is generally assumed that sex differences in physiology are negligible [1]. Additionally, female mice of various strains are known to show changes in physiology and behavior across the estrous cycle [2-4]. These factors have created a perception that females are more variable and therefore more difficult to study than males, leading researchers to neglect females to focus on males with findings generalized to females of

\footnotetext{
* Correspondence: kriegsfeld@berkeley.edu

${ }^{1}$ Department of Psychology, University of California, Berkeley, CA 94720, USA ${ }^{4}$ The Helen Wills Neuroscience Institute, University of California, Berkeley, CA 94720, USA

Full list of author information is available at the end of the article
}

the same species [5]. This male bias has not only led to false assumptions regarding female physiology and behavior but has also been harmful. For example, cancer chronotherapies developed in studies of men revealed that treatments at a particular circadian phase increased survival rates but that the same treatment regimen significantly decreased female survival $[6,7]$. Until recently, whether females are actually more variable than males has not been empirically investigated [8]. In a metaanalysis of 293 articles, the variance of behavioral, morphological, physiological, and molecular measures was not significantly greater in female than male mice for any parameter and was substantially greater in males for 
several traits [9]. A similar analysis of rats (311 articles) yielded similar results [10].

While one source of female variability likely results from hormonal fluctuations during the ovulatory cycle, the source and pattern of male variability has not been systematically characterized. We sought to examine sex differences in two widely used and widely applicable modalities: locomotor activity (LA) and core body temperature (CBT). LA and CBT are commonly used as proxies for physiological and neurological outputs in biology. In rodents, for example, daily changes in LA and CBT are associated with the progression of the estrous cycle [11-14]. The progressive delay in the offset of daily LA from the day of estrus in Syrian hamsters [14] and a corresponding delay in the daily decline of CBT on the day of estrus in C57 mice [13] point to the utility of LA and CBT in tracking physiologically relevant events and sources of variation.

Stereotyped changes in LA or CBT over both the day and the estrous cycle demonstrate that these modalities are structured across multiple timescales and that this temporal structure explains much of the variability in these data. To investigate potential sex differences at different timescales, we used wavelet transformation (WT) of LA and CBT data. Wavelets possess position, frequency, and amplitude information and are ideally suited for analysis of overlapping cycles occurring in the same data at different time scales. WTs have been successfully applied to studies of circadian biology to capture changes in power by periodicity across time [15-17], underscoring the efficacy of this approach. By uncovering sex differences and temporal structure in variability across timescales, studies can be designed to effectively consider these variables. Additionally, as LA and CBT effectively mirror underlying physiological change, identifying predictable patterns of variability sets the stage for further exploration of rhythmic patterns in underlying biological systems [18-22].

\section{Methods}

\section{Animals}

Data were analyzed from 13 male and 13 female BALB/c mice ( $>60$ days of age) in accordance with protocols approved by the Animal Care and Use Committee at UC Berkeley and in conformance with principles enunciated in the NIH Guide for the care and use of laboratory animals. Mice were housed under an LD 12:12 photocycle with ad libitum access to water and chow. Light onset and offset occurred at 0600 and $1800 \mathrm{~h}$, respectively. Humidity and temperature were held constant at $40 \%$ and $21{ }^{\circ} \mathrm{C}$, respectively.

\section{Core body temperature and locomotor activity data collection}

Data were gathered with Mini Mitter G2 E-Mitter implants that chronically record LA and CBT (Starr Life Sciences Co., Oakmont, PA). G2 E-Mitters were implanted in the intraperitoneal cavity (under isoflurane anesthesia, with analgesia achieved by subcutaneous injections of $0.03 \mathrm{mg} / \mathrm{kg}$ buprenorphine in saline, administered every $12 \mathrm{~h}$ for 2 days after surgery). E-Mitters were sutured to the ventral muscle wall to maintain consistent core temperature measurements. Recordings began immediately, but data collected for the first week postsurgery were not included in analyses. Recordings were continuous and stored in 1-min bins. Implants were placed in 7- to 10-week-old mice that were handled once per week during recordings (at the time of cage changes) but otherwise left undisturbed in single housing. Light intensity during the photo- and scotophases were $\sim 400$ lux white light and $<1$ lux red light, respectively.

\section{Data analysis}

Twelve days of data from each mouse were selected for analysis. In females, these days were aligned to a day of estrus, defined by the presence of an extended period of maximum CBT [13]. Thus, the 12 days encompassed three consecutive 4-day estrous cycles, with the first, fifth, and ninth days demonstrating an estrus-like profile characterized by a prolonged plateau of peak CBT, as previously reported [13]. For males, 12 consecutive days of data were chosen. For CBT, values below $35{ }^{\circ} \mathrm{C}$ were set to $35{ }^{\circ} \mathrm{C}$ to remove the result of a few rare device malfunctions and all values more than three standard deviations from the mean were set to three standard deviations from the mean. For LA, the correction to three standard deviations was only applied in the positive direction so that erroneously high values were corrected while activity counts of " 0 " were not inflated. The output from the G2 implant is in the form of degree Celsius and activity counts per unit time (events per $1 \mathrm{~min}$ ).

Daily range for each modality was defined as (maxmin) per mouse per day. Median 4-day windows (i.e., cycles in females) were generated for each animal by taking the average of the three repeated cycles, followed by taking the average of these 4-day windows across individuals of the same sex. Inter-animal variability was defined as the population range for each modality's daily range.

Data were analyzed and plotted using Matlab 2015b and 2016a in conjunction with in-house code for wavelet decomposition modified from the "Jlab" toolbox and from code developed by Prof. Tanya Leise [15], using the Morse wavelet (Morse parameters of $\beta=5$ and $\gamma=3$ that describe the frequencies of the two waves superimposed to create the wavelet) (see [23] for further description). 
Briefly, whereas Fourier transforms allow transformation of a signal into frequency space without temporal position (i.e., using sine wave components with infinite length), wavelets are constructed with amplitude diminishing to 0 in both directions from center. This property permits frequency strength calculation at a given position. Wavelets can assume many functions (shapes, e.g., Mexican hat, square wave, Morse); here, we use a Morse wavelet with relatively low number of oscillations (defined by $\beta$ and $\gamma$ ), similar to wavelets used in previous circadian applications $[15,24]$. This low number of oscillations enhances detection of contrast and transitions. Additional values of $\beta$ (3-8) and $\gamma(2-5)$ did not alter the findings. Because WTs exhibit artifacts at the edges of the data being transformed, WTs were performed on 14-day windows for each animal, with the 12 days previously analyzed, buffered by 1 day preceding and 1 day following. To avoid erroneous edge effects, only the WT of the 12 days analyzed previously were analyzed further. Periods of 1 to $39 \mathrm{~h}$ were assessed. For statistical comparisons of populations, Wilcoxon rank sum tests were used to avoid assumptions of normality for any distribution; note that degrees of freedom are not used in Wilcoxon rank sum tests. Non-parametric Kruskal-Wallis tests were used instead of ANOVAs for the same reason; for all Kruskal-Wallis tests, $\chi^{2}$ and $p$ values are listed in the text and all such comparisons have the same $N /$ group, and so the same degrees of freedom $(\mathrm{df}=\mathrm{N} /$ group $-1=12$ ). For quantification of spectral differences, WT spectra were isolated in two 2-h bands; circadian periodicity power was defined as the max power per minute within the 23-25-h band; ultradian periodicity power was defined as the max power per minute in the 1-3-h band. The latter band was chosen because this band corresponded with the daily ultradian peak power observed in ultradian rhythms (URs) across physiological systems [21, 22, 24-31]. A color map for wavelets was developed by our group to provide red-green colorblindcompliant uniform contrast across the range of data, with the exception of the two extremes (highest and lowest $10 \%$ ), which are brightened to highlight extreme high and low values. Figures were formatted in Microsoft PowerPoint 2013 and Adobe Photoshop CS6.

\section{Results}

As expected, females exhibited a 4-day cycle in LA and CBT (Fig. 1a, c). Females show a prolonged peak of daily LA and CBT on the day of estrus, as described previously $[13,14]$, while males do not exhibit an obvious 4day cycle. Within-animal daily range was significantly greater in males for CBT (Fig. $1 \mathrm{~d} ; p<1 \times 10^{-4}$ ) and LA (Fig. $1 \mathrm{~b} ; p<1 \times 10^{-4}$ ). Between-animal variability was greater for males in CBT and comparable to females in LA. (Fig. 1b, d; bars extending from each box; CBT:

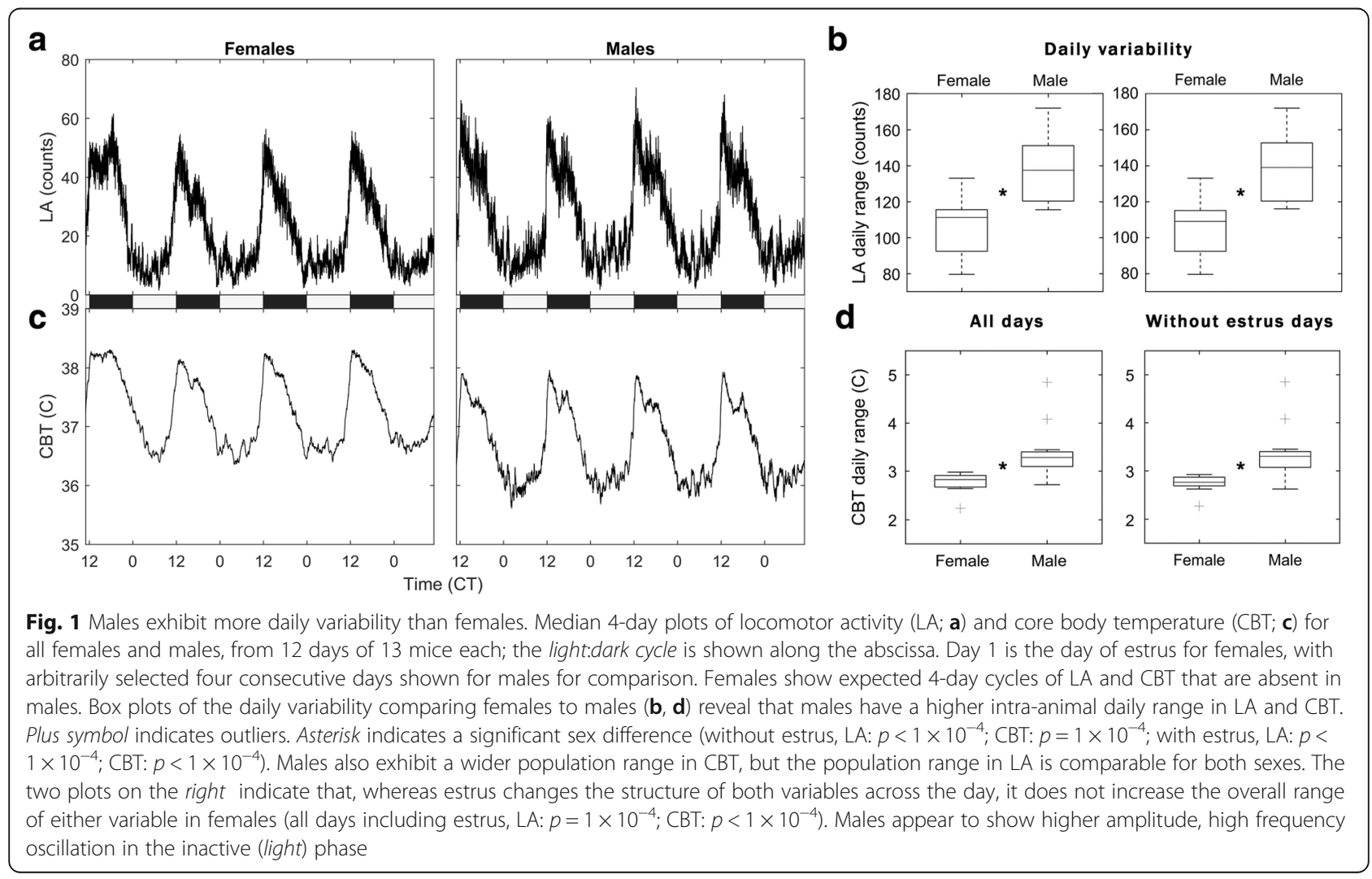


$R_{\text {male }}=2.12, R_{\text {fem }}=0.75 ;$ LA: $\left.R_{\text {male }}=56.34, R_{\mathrm{fem}}=53.49\right)$. To assess whether or not estrus increased the inter- or intra-individual range of female LA or CBT, we reanalyzed these daily ranges excluding days of estrus (i.e., for parity, excluding days 1, 5, and 9 from the analysis in both males and females). As predicted, estrus did not substantially change either the inter- or intraindividual ranges in these parameters, nor did it enhance the differences across sexes observed with all days included (Fig. 1b, d, left vs. right; CBT: $p<1 \times$ $10^{-4}$ without estrus vs. $1 \times 10^{-4}$ with all days; LA: $p$ $=1 \times 10^{-4}$ without estrus vs. $\left.p=1 \times 10^{-4}\right)$. This is not to imply that estrus does not represent a source of infradian variance in females; rather, that the day of estrus does not increase the daily variability of female LA or CBT. Instead, estrus manifests as a change in the shape of LA and CBT across the day, with a longer plateau of peak temperature and activity during the active phase than on non-estrous days.

WT analysis reveals sex differences in LA and CBT at the ultradian and infradian, but not circadian periodicity. Neither sex exhibits an effect of day at the circadian periodicity $(23-25 \mathrm{~h})$ for either LA $\left(\chi^{2}=3.64, p>0.05\right.$, $X^{2}=0.37, p>0.05$ for females and males, respectively) or CBT $\left(\chi^{2}=0.85, p>0.05, \chi^{2}=0.68, p>0.05\right.$ for females and males, respectively) (Fig. 2a-g). Female infradian rhythms manifest as a significant reduction in 1-3-h ultradian power specifically on the day of estrus as compared to the ultradian power in any of the subsequent 3 days (Fig. $2 \mathrm{~h}, \mathrm{i})\left(\chi^{2}=13.52, p<1 \times\right.$ $\left.10^{-3}\right)$. Males have significantly higher power $1-3-\mathrm{h}$ URs in LA than females (Fig. 2h) $\left(\chi^{2}=34.36, p<1 \times\right.$ $10^{-8}$ ). Furthermore, males exhibit a change from dark to light phase CBT UR power nearly twice that of females (Fig. 2i) $\left(\chi^{2}=54.83, p<1 \times 10^{-12}\right.$ for males; $\chi^{2}=30.61, p<1 \times 10^{-7}$ for females). Females are more variable than males on the infradian scale, but males are more variable than females on an ultradian scale.

\section{Discussion}

Female rodents have long been discounted as study subjects because estrous cycles are assumed to generate greater variability across traits than is seen in males. Until recently, substantive data to support or refute this conjecture were lacking. Two metaanalyses now have demonstrated for mice [9] and rats [10] that females tested at random stages of the estrous cycle are no more variable than males for numerous traits, consistent with genetic profiling arrays [32]; similar data have been obtained for unstaged human subjects [28]. The source of variability in males over the course of 4 days corresponding to the female estrous cycle has not been examined prior to the present study. Contrary to earlier assumptions, we found that median circadian power is comparable between the sexes. Whereas females exhibit infradian rhythms associated with the estrous cycle, males show significantly greater UR power than females, suggesting that differences in timescales of biological rhythms may help explain overall similarity in variability between the sexes $[9,10]$. Stated differently, females vary across infradian cycles of 4 days but males are more variable than females within a single day. These findings underscore that males should not necessarily be favored over females in rodent studies and point to sex differences across timescales as worthy of explicit exploration in systems of interest.

To understand the significance of sex differences in ultradian power, it is necessary to understand the physiological dynamics underlying CBT temporal structure. URs have not been extensively investigated, but several candidate systems both exhibit URs in the $1-3-h$ range and modulate CBT. These systems include the hypothalamo-pituitary-gonadal axis [13, 18-20, 33], the hypothalamo-pituitary-adrenal axis [21, 24-29, $34,35]$, and the suprachiasmatic nucleus [30]. The central dopaminergic axis exhibits URs in the 1-3-h range [31] and may influence CBT as well through modulation of activity and appetitive behaviors, like eating and drinking. If each of these systems independently modifies CBT, one would expect to see a peak of ultradian power for each system. However, our data show a relative consolidation of ultradian frequencies, consistent with a framework in which these distinct physiological systems belong to a network of coupled oscillators. Oscillators that couple eventually drive each other to synchrony [36]. Physiologically, some evidence already exists for coupling across these systems [37]. For example, luteinizing hormone ( $\mathrm{LH})$, a pituitary peptide well known for regulating reproductive axis functioning, also affects steroid hormone production in the adrenals [38]. In turn, adrenal steroids can feed back to the brain to inhibit LH $[39,40]$. However, the physical extent and the temporal persistence of this hypothetical-coupled network within an individual have not been systematically studied.

As relationships connecting LA and CBT rhythms with more difficult-to-measure endpoints are described, this knowledge can be used to design studies that eliminate time-consuming, disruptive assays such as repeated blood collection [41] or to estimate outcome measures in between collection points to add greater effective temporal resolution. If CBT rhythms reflect changes in several physiological systems, then males may show larger variability than females across those systems at the ultradian time scale (e.g., higher amplitude change in URs of sex-hormone release, feeding). Whether the 

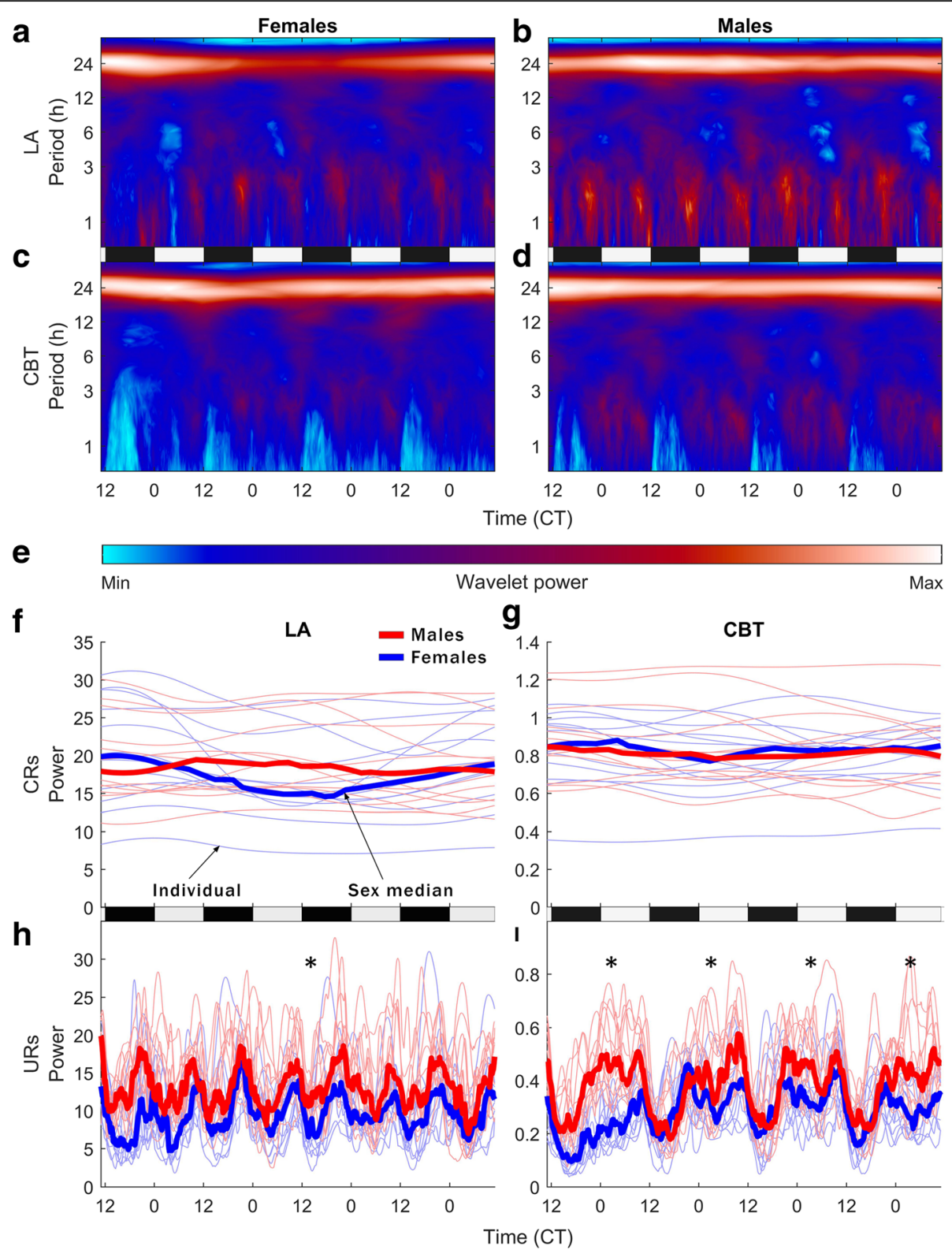

Fig. 2 Median wavelet transforms (WTs; a-d) of the data that comprise the average depicted in Fig. 1 indicate changes in rhythmic power (e) across periodicities ranging from 1 to $39 \mathrm{~h}$ (log $\mathrm{y}$-axis). Day 1 is the day of estrus for females $(\mathbf{a}, \mathbf{c})$ and an arbitrary four consecutive days chosen for males $(\mathbf{b}, \mathbf{d})$. Females show an effect of day in ultradian CBT, the depression of which identifies estrus $\left(X^{2}=13.52, p<5 \times 10^{-3}\right)$. Males do not show an effect of day but exhibit greater power in high frequency $\left(1-3-h\right.$ periodicity) URs in $L A\left(x^{2}=34.36, p=1 \times 10^{-8}\right)$. Quantification of individual and median circadian power (CRs, 23-25-h) (f, $\mathbf{g})$ and ultradian power (URs, 1-3-h) (h, i). For CBT, male UR power is greater only during the inactive phase (i). Asterisk indicates a significant sex difference. Both sexes show an effect of time of day for URs in CBT, but males have nearly twice the ultradian change of females $\left(x^{2}=54.83, p<1 \times 10^{-12}\right.$ for males; $x^{2}=30.61, p<1 \times 10^{-7}$ for females). No sex difference is detectable in the circadian range (23-25-h)

present findings generalize to rats or other rodents that undergo estrous cycles is presently unknown and subject to experimental verification. A range of factors affecting temporal structure in addition to sex (e.g., age, strain, species) warrant exploration using similar approaches so that a maximum variance in physiological measures can be accounted for.

\section{Conclusions}

This study confirms that male BALB/c mice show greater variability across the day, while females show an infradian rhythm that males do not. Our finding of significant sex differences in ultradian power suggests that inclusion of female mice is advantageous where observations are made over the course of several hours-in 
contrast to current practice, which generally excludes female mice from many experiments [9]; because male ultradian variability exceeds that of females, females are more likely to provide consistent results when measures are obtained over the course of several hours. Finally, whereas the mechanisms that generate URs in CBT remain unknown, the difference in UR power points to basic differences in male and female physiology. Investigating URs in both sexes in tandem may provide novel insights into sex differences in physiology and behavior. In light of the many sex differences in human biology, studying both sexes will increase the translational impact of mouse research, helping to avoid inappropriate generalization of findings from males to females.

\section{Abbreviations}

CBT: Core body temperature; LA: Locomotor activity; UR: Ultradian rhythm; WT: Wavelet transformation

\section{Acknowledgements}

The authors wish to thank Prof. Tanya Leise at Amherst College for kindly sharing her wavelet analysis code, which our code is modified from, and Prof. Annaliese Beery at Smith College for her thoughts on the initial experiments.

\section{Funding}

This work was funded by the National Institute of Child Health and Human Development-F32 HD081957 (BS) and HD050470 (LJK).

\section{Availability of data and materials}

All data and code used for analyses in this manuscript are available in the supplemental materials.

\section{Authors' contributions}

BS contributed to the design of the experiments and executed the experiments and data analysis. BS also generated all figures and code and contributed to the writing of the manuscript. AG contributed to the execution of the experiment and assisted in data analysis and figure design. She also contributed to the writing of the manuscript. IZ, BP, and LK contributed to experimental design and writing of the manuscript. LJK additionally provided materials and infrastructure to enable the experiments. All authors contributed to the writing of the manuscript and gave final approval of the published version. All authors are accountable for all aspects of the work.

\section{Competing interests}

The authors declare that they have no competing interests.

\section{Consent for publication}

Not applicable

\section{Ethics approval}

All work was carried out in accordance with protocols approved by the Animal Care and Use Committee at UC Berkeley and in conformance with principles enunciated in the NIH Guide for the care and use of laboratory animals.

\section{Author details}

'Department of Psychology, University of California, Berkeley, CA 94720, USA. ${ }^{2}$ Department of Integrative Biology, University of California, Berkeley, CA, USA. ${ }^{3}$ Department of Psychology, University of Chicago, Chicago, IL, USA. ${ }^{4}$ The Helen Wills Neuroscience Institute, University of California, Berkeley, CA 94720, USA.

Received: 4 November 2016 Accepted: 26 December 2016 Published online: 09 February 2017

\section{References}

1. Institute of Medicine (US) Committee on understanding the biology of sex and gender differences. Exploring the biological contributions to human health: does sex matter? eds Wizemann TM, Pardue M-L (National Academies Press (US), Washington (DC)). 2001. Available at: http://www. ncbi.nlm.nih.gov/books/NBK222288/. Accessed April 27, 2016.

2. Barthelemy M, Gourbal BEF, Gabrion C, Petit G. Influence of the female sexual cycle on BALB/C mouse calling behaviour during mating. Naturwissenschaften. 2004;91(3):135-8.

3. Meziane H, Ouagazzal A-M, Aubert L, Wietrzych M, Krezel W. Estrous cycle effects on behavior of C57BL/6J and BALB/CByJ female mice: implications for phenotyping strategies. Genes Brain Behav. 2007;6(2):192-200.

4. Krzych U, Strausser HR, Bressler JP, Goldstein AL. Quantitative differences in immune responses during the various stages of the estrous cycle in female BALB/c mice. J Immunol Baltim Md. 1978;121(4):1603-5.

5. Hughes RN. Sex does matter: comments on the prevalence of male-only investigations of drug effects on rodent behaviour. Behav Pharmacol. 2007;18(7):583-9.

6. Giacchetti S, et al. Phase III trial comparing 4-day chronomodulated therapy versus 2-day conventional delivery of fluorouracil, leucovorin, and oxaliplatin as first-line chemotherapy of metastatic colorectal cancer: the European Organisation for Research and Treatment of Cancer Chronotherapy Group. J Clin Oncol Off J Am Soc Clin Oncol. 2006;24(22):3562-9.

7. Lévi F, Okyar A, Dulong S, Innominato PF, Clairambault J. Circadian timing in cancer treatments. Annu Rev Pharmacol Toxicol. 2010;50:377-421.

8. Beery AK, Zucker I. Sex bias in neuroscience and biomedical research. Neurosci Biobehav Rev. 2011;35(3):565-72.

9. Prendergast BJ, Onishi KG, Zucker I. Female mice liberated for inclusion in neuroscience and biomedical research. Neurosci Biobehav Rev. 2014;40:1-5.

10. Becker JB, Prendergast BJ, Liang JW. Female rats are not more variable than male rats: a meta-analysis of neuroscience studies. Biol Sex Differ. 2016;7:34.

11. Kopp C, Ressel V, Wigger E, Tobler I. Influence of estrus cycle and ageing on activity patterns in two inbred mouse strains. Behav Brain Res. 2006;167(1):165-74.

12. Basterfield L, Lumley LK, Mathers JC. Wheel running in female C57BL/6J mice: impact of oestrus and dietary fat and effects on sleep and body mass. Int J Obes. 2009;33(2):212-8.

13. Sanchez-Alavez M, Alboni S, Conti B. Sex- and age-specific differences in core body temperature of C57BI/6 mice. Age Dordr Neth. 2011;33(1):89-99.

14. Prendergast BJ, Beery AK, Paul MJ, Zucker I. Enhancement and suppression of ultradian and circadian rhythms across the female hamster reproductive cycle. J Biol Rhythms. 2012;27(3):246-56.

15. Leise TL, Harrington ME. Wavelet-based time series analysis of circadian rhythms. J Biol Rhythms. 2011;26(5):454-63.

16. Leise TL, Indic P, Paul MJ, Schwartz WJ. Wavelet meets actogram. J Biol Rhythms. 2013;28(1):62-8.

17. Leise TL. Wavelet analysis of circadian and ultradian behavioral rhythms. J Circadian Rhythms. 2013;11(1):5.

18. Mauvais-Jarvis F. Estrogen and androgen receptors: regulators of fuel homeostasis and emerging targets for diabetes and obesity. Trends Endocrinol Metab TEM. 2011;22(1):24-33.

19. Marrone BL, Thomas Gentry R, Wade GN. Gonadal hormones and body temperature in rats: effects of estrous cycles, castration and steroid replacement. Physiol Behav. 1976;17(3):419-25.

20. Rance NE, Dacks PA, Mittelman-Smith MA, Romanovsky AA, Krajewski-Hall SJ. Modulation of body temperature and LH secretion by hypothalamic KNDy (kisspeptin, neurokinin B and dynorphin) neurons: a novel hypothesis on the mechanism of hot flushes. Front Neuroendocrinol. 2013;34(3):211-27.

21. Sanchez-Alavez $M$, et al. Insulin causes hyperthermia by direct inhibition of warm-sensitive neurons. Diabetes. 2010;59(1):43-50.

22. Weinert $D$, Waterhouse J. The circadian rhythm of core temperature: effects of physical activity and aging. Physiol Behav. 2007;90(2-3):246-56.

23. Lilly JM, Olhede SC. Generalized Morse wavelets as a superfamily of analytic wavelets. IEEE Trans Signal Process. 2012;60(11):6036-41.

24. Smarr BL, Zucker I, Kriegsfeld L. Detection of successful and unsuccessful pregnancies in mice within hours of pairing through frequency analysis of high temporal resolution core body temperature data. PLoS One. 2016;11(7):e0160127.

25. Qian X, Droste SK, Lightman SL, Reul JMHM, Linthorst ACE. Circadian and ultradian rhythms of free glucocorticoid hormone are highly synchronized between the blood, the subcutaneous tissue, and the brain. Endocrinology. 2012;153(9):4346-53. 
26. Wang ZY, Cable EJ, Zucker I, Prendergast BJ. Pregnancy-induced changes in ultradian rhythms persist in circadian arrhythmic Siberian hamsters. Horm Behav. 2014;66(2):228-37.

27. Fulkerson WJ, Tang BY. Ultradian and circadian rhythms in the plasma concentration of cortisol in sheep. J Endocrinol. 1979;81(1):135-41.

28. Smarr B, Burnett D, Mesri S, Pister K, Kriegsfeld L. A wearable sensor system with circadian rhythm stability estimation for prototyping biomedical studies. IEEE Trans Affect Comput PP. 2015;99:1-1.

29. Young EA, Abelson J, Lightman SL. Cortisol pulsatility and its role in stress regulation and health. Front Neuroendocrinol. 2004;25(2):69-76.

30. Ono D, Honma K, Honma S. Circadian and ultradian rhythms of clock gene expression in the suprachiasmatic nucleus of freely moving mice. Sci Rep. 2015:5:12310.

31. Blum ID, et al. A highly tunable dopaminergic oscillator generates ultradian rhythms of behavioral arousal. eLife. 2014:3:e05105. doi:10.7554/eLife.05105.

32. Itoh Y, Arnold AP. Are females more variable than males in gene expression? Meta-analysis of microarray datasets. Biol Sex Differ. 2015;6:18.

33. de la Iglesia HO, Schwartz WJ. Minireview: timely ovulation: circadian regulation of the female hypothalamo-pituitary-gonadal axis. Endocrinology. 2006;147(3):1148-53

34. Plat $L$, et al. Effects of morning cortisol elevation on insulin secretion and glucose regulation in humans. Am J Physiol. 1996;270(1 Pt 1):E36-42.

35. Lambillotte C, Gilon P, Henquin JC. Direct glucocorticoid inhibition of insulin secretion. An in vitro study of dexamethasone effects in mouse islets. J Clin Invest. 1997:99(3):414-23.

36. Mallada E, Tang A. Synchronization of weakly coupled oscillators: coupling, delay and topology. J Phys Math Theor. 2013;46(50):505101.

37. Cardon SZ, Iberall AS. Oscillations in biological systems. Curr Mod Biol. 1970; 3(3):237-49.

38. Kero J, et al. Elevated luteinizing hormone induces expression of its receptor and promotes steroidogenesis in the adrenal cortex. J Clin Invest. 2000;105(5):633-41.

39. Williams NI, Berga SL, Cameron JL. Synergism between psychosocial and metabolic stressors: impact on reproductive function in cynomolgus monkeys. Am J Physiol Endocrinol Metab. 2007;293(1):E270-6.

40. Battaglia DF, Krasa HB, Padmanabhan V, Viguié C, Karsch FJ. Endocrine alterations that underlie endotoxin-induced disruption of the follicular phase in ewes. Biol Reprod. 2000;62(1):45-53.

41. Tsai P-P, et al. Effects of different blood collection methods on indicators of welfare in mice. Lab Anim. 2015;44(8):301-10.

\section{Submit your next manuscript to BioMed Central and we will help you at every step:}

- We accept pre-submission inquiries

- Our selector tool helps you to find the most relevant journal

- We provide round the clock customer support

- Convenient online submission

- Thorough peer review

- Inclusion in PubMed and all major indexing services

- Maximum visibility for your research

Submit your manuscript at www.biomedcentral.com/submit

) Biomed Central 